\title{
Tribute for Takayuki Tamura on his 90th birthday
}

\section{Howard B. Hamilton • Thomas E. Nordahl}

Received: 8 April 2009 / Published online: 11 June 2009

(C) Springer Science+Business Media, LLC 2009

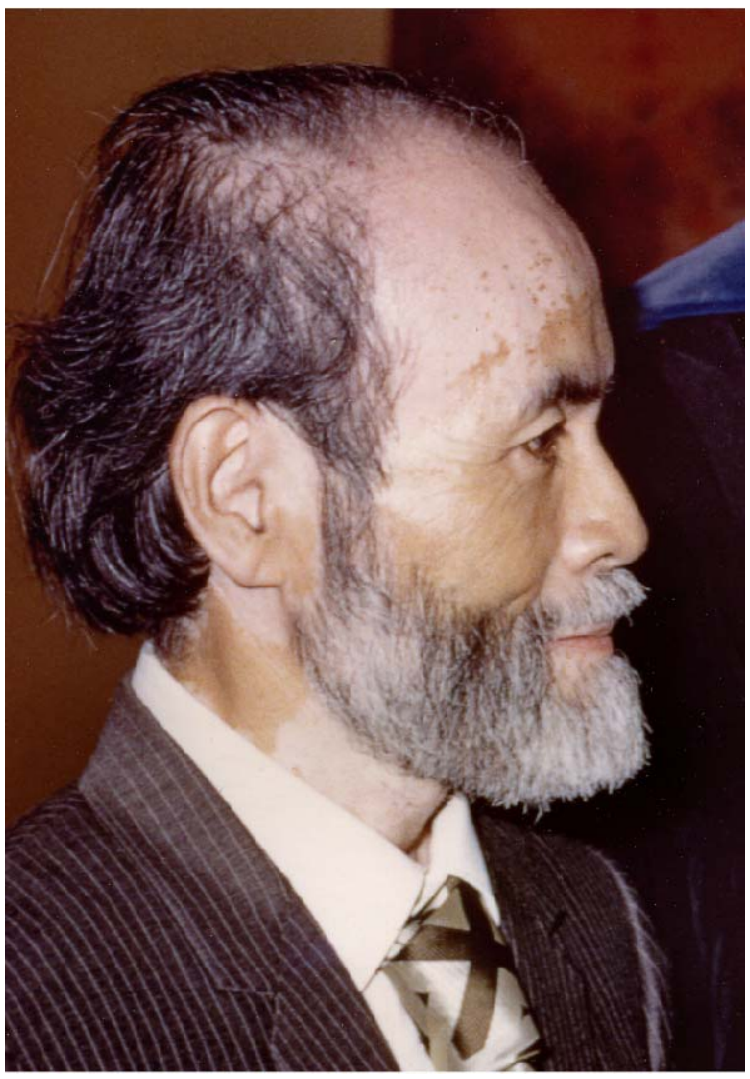

Communicated by László Márki.

H.B. Hamilton

Department of Mathematics, California State University at Sacramento, 6000 J St., Sacramento, CA, USA

e-mail: hamiltonhatma@msn.com

T.E. Nordahl $(\bowtie)$

Department of Psychiatry and Behavioral Sciences, University of California at Davis, 2230 Stockton Blvd., Sacramento, CA, USA

e-mail: tenordahl@ucdavis.edu 
Takayuki Tamura was born in Tokushima City, Japan on March 12, 1919; he was raised in a British-Style house with hipped roof, as he would say. Tamura's parents always encouraged him in his interest in mathematics and he greatly appreciated them. He met his future wife, Kimie, when she was 5 years old in 1928. He and Kimie were married on December 31, 1945. She was a good complement and partner to Takayuki until her recent death. In traditional Japanese style she would walk 3 steps behind him. Their daughter, Hiroko, was born on October 31, 1946. Hiroko studied art at an art school in San Francisco and has continued her art work.

Tamura's education started with his elementary schooling at Tokushima City Middle School in Tokushima City, Japan. Next he attended Tokyo Science College from 1939 to 1942 . This was followed by a stint from 1942 to 1944 at Osaka Imperial University. Tamura taught at Tokushima Middle School in 1946. Then he received an academic appointment at Tokushima Youth Normal College as Professor from 1946 to 1960 . He, also, studied individually with his advisor Kenjiro Shoda. He published papers until it was felt that he had published sufficiently many works and he was granted a Doctor of Science degree from the Osaka Imperial University in 1958. Tamura referred to this as the "Paper-Doctor System".

On December 7, 1941 Tamura was 22 years old and at 4'10" $(=147 \mathrm{~cm})$; he had been rejected for military service as being too short. He would say that he was glad that he did not have to fight the United States. On the night of July 4, 1945 Tokushima City was reduced to ashes by an aerial bombing by US forces. All of his family's property was lost during that one night, just one month before WWII was over. His parents were older than 60 then and Takayuki did not live in Tokushima at that time but was working at Osaka Imperial University. It is perhaps not a big surprise that his and Kimie's families were concerned when Tamura and his immediate family moved to the United States in 1960. Takayuki Tamura, his wife Kimie and his then 14 year-old daughter, Hiroko, came to Davis in 1960 when the UC Davis Mathematics Department began to offer the Ph.D. degree.

In the late 60's the authors of this note were undergraduate students at UCD and one of the graduate students, Ed Shoemaker, befriended us and encouraged us to take a class from Tamura while he was still alive. With his gray-white beard it was hard for us twenty-year olds to judge his age. Tamura, as we would learn later, was only approaching 50 years of age. We began taking his algebra class and his seminars and began the gradual evolution in becoming his students without officially asking.

Tamura's speech was not always clear, Japanese speaking individuals told us that they also had some trouble understanding him as he had a lisp. He was clearly a warm-hearted person and handed us beautifully penned, nearly verbatim copies for each lecture that he would deliver.

Tamura and Kimie delighted in having students over to their place in Davis. We were regaled with their stories of being surprised on their first Halloween in the United States, wondering what these children dressed up in costumes were up to. Kimie would show off her origami work. For one party, a formal tea ceremony which we attended with our spouses, Kimie would keep close eye on us and ensure that we were sitting appropriately for the ceremony. We were privileged to attend the Buddhist wedding of their beautiful daughter, Hiroko, to Junji, a biochemist, who took a faculty position in Louisiana. The ceremony was held at Tamura's house and was 
an interesting contrast as the Buddhist priest was Caucasian. Such an event was rarer then than it would be now.

Tamura would pose for pictures at some of the parties at his house by standing on his tiptoes, or by standing on the base of the fireplace. We would be taught the importance of honoring our mathematical ancestors. We would learn that his teacher, Kenjiro Shoda, had studied with the famous Emmy Noether at Göttingen and that Shoda had subsequently received the Culture Merit Award of Japan for bringing modern mathematics to Japan. Shoda would tell Tamura and his students about how every day was alive with Noether bringing in fresh new work or fresh approaches to old work. Tamura gave us the strict proscription not to let Emmy Noether down. His great wish was that we would be excellent teachers and that we would not disappoint our teacher ancestor, Emmy Noether. Tamura would refer to Shoda as our "grand teacher" and Noether as our "great grand teacher". In developing mathematics we were told to take on topics that were initially not popular and make them popular.

Through our graduate years we were privileged to meet a number of the leading researchers in semigroup theory who would come to visit and present at Tamura's algebra seminar or else to the department at large. When AH Clifford came to speak to the Department it was a quite special occasion as this was the Clifford of Clifford and Preston, the first and main English Semigroup Text, the bible. Clifford, ever the gentleman, began alluding to Tamura that to speak of semilattice decomposition at UC Davis was like bringing coals to Newcastle.

The American Mathematical Society's MathSciNet lists 166 papers of Dr. T. Tamura (not counting supplements and errata separately; it actually lists 2 more but those on quantum physics are not his). In addition, he has written a book on semigroups in Japanese. Tamura

(1) began the study of greatest homomorphic image of a type $T$, where $T$ is a collection of identities;

(2) established the finding that every semigroup is a semilattice union of semilatticeindecomposable subsemigroups and observed further with Kimura that if $S$ were commutative, these semilattice-indecomposable subsemigroups would be Archimedean;

(3) characterized the structure of $N$-semigroups, that is, commutative cancellative Archimedean semigroups without idempotents and showed that all $\mathrm{N}$ semigroups have a proper homomorphism into the non-negative reals under the operation of addition;

(4) established that the set of identities $\{x x=x, x y=y x\}$ is the only proper set of identities, $T$, on the class of semigroups that give a $T$-decomposition into a union of $T$-indecomposable subsemigroups (attainability).

Arguably, this result explains to a large extent why semilattice decompositions play such a prominent role in the structure theory of semigroups. Boris Schein noted that when A.I. Malcev discussed the attainability results of Tamura it was the first time that Malcev had ever discussed the topic of semigroups. Malcev extended the results of Tamura on attainability and defined a multiplication (now called Malcev product) for classes of algebraic systems that is of interest to researchers in universal algebra. 
Early in his career Tamura had published a list of all semigroups of order 3, 4, and 5 up to isomorphism and anti-isomorphism. This was done by hand with some help from his students in Japan. Takayuki Tamura's last work involved studying the power semigroup, $P(S)$, of a semigroup. He was an initiator of the study of power semigroups of a semigroup, testing to see if $S$ is isomorphic to $T$ when $P(S)$ is isomorphic to $P(T)$. This question was answered in the negative for infinite semigroups by Mogiljanskaja, but Tamura was determined to show it had an affirmative answer for finite semigroups. One of the current paper's authors was still attending Tamura's Tuesday afternoon seminars during the 1980's and Tamura would show up most weeks with a new typed copy of his recent work on power semigroups. We would go over this new work with him and the complexity of it all was overwhelming to many of us. To see Dr. Tamura's ability to easily handle the vast amounts of such complicated structure was to this author the most amazing exhibition of Tamura's creative talent and his powerful mind. This author had a conversation with Peter Jones at a conference on semigroups at which Peter told him that Tamura was sending him copy after copy of this power semigroup stuff and asked whether he should be reading it all or should wait until Tamura had the final results. The isomorphism problem for finite semigroups remains open. The study of power semigroups has subsequently become of interest to those who study pseudovarieties. Researchers would be interested in problems including the study of (pseudo)varieties closed under the power set operation.

A major work for Tamura involved his help in teaching more than 20 students who achieved their Ph.Ds. under his guidance at UC Davis and while he was on the faculty of Tokushima Youth Normal College from 1946-1960. A perusal of his papers might give one a good idea of his students as he published many papers with co-authors including students. His students, besides the authors, also included James Chrislock and Junitaka Shoji. Tamura had great hopes for Kenya Yamaoka solving long-standing "historic problems", but his father required Kenya to take over the family business and forbade all mathematical contact with him.

Tamura's favorite idea/paper was his main work on the attainability of identities. He presented this at the International Congress of Mathematicians in Moscow, 1966 and it was published in the Journal of Algebra. He enjoyed the excitement of being at this congress. It was his favorite conference of all of the ones he attended.

He is not working on any mathematics now. He mentioned collecting students' papers. He also is collecting Japanese and English poetry and is a member of Short Poems of America and the Meiji Shrine Short Poem Society of Japan.

He was walking regularly to the UCD Math Department for many years till December of 2008. He says now that it is too far for him to walk and he is no longer walking the several miles to the Department.

When Tamura was asked at his 90th year birthday brunch what he had learned during his 90 years, he replied: "A miracle is my life".

\section{List of publications of Takayuki Tamura}

1. Characterization of groupoids and semilattices by ideals in a semigroup, J. Sci. Gakugei Fac. Tokushima Univ. 1 (1950), 37-44. 
2. On a relation between local convexity and entire convexity, J. Sci. Gakugei Fac. Tokushima Univ. 1 (1950), 25-30.

3. On the system of semigroup operations defined in a set, J. Gakugei Coll. Tokushima Univ. 2 (1952), 1-12.

4. Remarks on the convexity of connected sets, J. Gakugei Tokushima Univ. 3 (1953), 24-27.

5. Some remarks on semi-groups and all types of semi-groups of order 2,3, J. Gakugei Tokushima Univ. 3 (1953), 1-11.

6. On finite one-idempotent semigroups, I., J. Gakugei Tokushima Univ. (Nat. Sci.) 4 (1954), 11-20.

7. On compact one-idempotent semi-groups \{Volume numbers not printed on issues until Vol. 7 (1955)\}, Kōdai Math. Sem. Rep. 6 (1954), 17-21; Supplement, ibid. 6 (1954), 96.

8. Note on unipotent inversible semigroups \{Volume numbers not printed on issues until Vol. 7 (1955)\}, Kōdai Math. Sem. Rep. 6 (1954), 93-95.

9. On decompositions of a commutative semigroup (with Kimura, Naoki), \{Volume numbers not printed on issues until Vol. 7 (1955)\}, Ködai Math. Sem. Rep. 6 (1954), 109-112.

10. On a monoid whose sobmonoids form a chain, J. Gakugei Tokushima Univ. Math. 5 (1954), 8-16.

11. Notes on finite semigroups and determination of semigroups of order 4, J. Gakugei Tokushima Univ. Math. 5 (1954), 17-27.

12. Counter examples to Wallace's problem (with Kimura, Naoki), Proc. Japan Acad. 31 (1955), 499-500.

13. All semigroups of order at most 5 (with Tetsuya, Kazutoshi; Hashimoto, Takao; Akazawa, Tadao; Shibata, Ryōichi; Inui, Tadashi), J. Gakugei Tokushima Univ. Nat. Sci. Math. 6 (1955), 19-39. Errata, on loose, unpaginated sheet.

14. One-sided bases and translations of a semigroup, Math. Japon. 3 (1955), 137141.

15. Existence of greatest decomposition of a semigroup (with Kimura, Naoki), Kōdai Math. Sem. Rep. 7 (1955), 83-84.

16. On translations of a semigroup, Kōdai Math. Sem. Rep. 7 (1955), 67-70.

17. Compact mob with a unique left unit (with Kimura, Naoki), Math. J. Okayama Univ. 5 (1956), 115-119.

18. Indecomposable completely simple semigroups except groups, Osaka Math. J. 8 (1956), 35-42; Errata, ibid. 9 (1957), 241.

19. The theory of construction of finite semigroups, I., Osaka Math. J. 8 (1956), 243-261; Errata, ibid. 9 (1957), 241.

20. On a special semilattice with a minimal condition, J. Gakugei Tokushima Univ. Nat. Sci. Math. 7 (1956), 9-17.

21. The theory of construction of finite semigroups, II., Osaka Math. J. 9 (1957), 1-42; Supplement, ibid. 9 (1957), 235-237; Errata, ibid. 242.

22. Commutative nonpotent Archimedean semigroup with cancelation law, I., J. Gakugei Tokushima Univ. 8 (1957), 5-11.

23. Distributive multiplications to semigroup operations (with Nakao, Mamoru; Shingai, Mitsuo; Iwano, Yasushi; Minami, Katsumi; Nii, Katsuyuki; Tateyama, 
Hiroshi), J. Gakugei Tokushima Univ. 8 (1957), 91-101; Errata, ibid. 9 (1957), 25.

24. Notes on translations of a semigroup, Kōdai Math. Sem. Rep. 10 (1958), 9-26.

25. The theory of construction of finite semigroups, III. Finite unipotent semigroups, Osaka Math. J. 10 (1958), 191-204.

26. Characterization of certain additive semigroups by distributive multiplications, J. Gakugei Tokushima Univ. Math. 9 (1958), 21-24.

27. Note on finite simple $c$-indecomposable semigroups, Proc. Japan Acad. 35 (1959), 13-15.

28. Finite semigroups in which Lagrange's theorem holds (with Sasaki, Morio), J. Gakugei Tokushima Univ. 10 (1959), 33-38.

29. Semigroups of order $\leq 10$ whose greatest $c$-homomorphic images are groups (with Sasaki, Morio; Minami, Yasuo; Noguchi, Toshio; Miki, Kenji; Shingai, Mitsuo; Nagaoka, Tsuguyo; Arai, Toshitaka; Muramoto, Katsuyo; Nakao, Mamoru; Maruo, Hiroaki; Himeda, Yoshiaki; Takami, Teruko), J. Gakugei Tokushima Univ. 10 (1959), 43-64.

30. Note on finite semigroups which satisfy certain group-like condition, Proc. Japan Acad. 36 (1960), 62-64.

31. Decompositions of a completely simple semigroup, Osaka Math. J. 12 (1960), 269-275.

32. Semigroups of order $5,6,7,8$ whose greatest $c$-homomorphic images are unipotent semigroups with groups (with Dehara, Kimiyoshi; Iwata, Tadao; Saito, Hiroyuki; Tsukumo, Keiji), J. Gakugei Tokushima Univ. 11 (1960), 5366.

33. Note on a semigroup having no proper subsemigroup, Proc. Japan Acad. 37 (1961), 72-74.

34. Note on the direct product of certain groupoids (with Merkel, R.B.; Latimer, J.F.), Proc. Japan Acad. 37 (1961), 482-484.

35. On semigroup whose subsemigroup semilattice is the Boolean algebra of all subsets of a set, J. Gakugei Tokushima Univ. 12 (1961), 1-3.

36. Certain characterization of singular semigroups, Bull. Amer. Math. Soc. 68 (1962), 402-404.

37. Note on $\Gamma^{*}$-semigroups, Bull. Amer. Math. Soc. 68 (1962), 505-508.

38. A note on the extension of semigroups with operators (with Burnell, Donald G.), Proc. Japan Acad. 38 (1962), 495-498.

39. The direct product of right singular semigroups and certain groupoids (with Merkel, Rudolph B.; Latimer, Jack F.), Proc. Amer. Math. Soc. 14 (1963), 118123; Correction and supplement, ibid. 16 (1965), 841.

40. Examples of direct products of semigroups or groupoids, Bull. Amer. Math. Soc. 69 (1963), 419-422.

41. Minimal commutative divisible semigroups, Bull. Amer. Math. Soc. 69 (1963), 713-716.

42. Semigroups and their subsemigroup lattices, Pacific J. Math. 13 (1963), 725736.

43. Semigroups of positive integer vectors (with Sasaki, Morio), Proc. Japan Acad. 39 (1963), 289-293.

44. Some special groupoids, Math. Japon. 8 (1963), 23-31. 
45. Operations on binary relations and their applications, Bull. Amer. Math. Soc. 70 (1964), 113-120.

46. Extension of groupoids with operators (with Burnell, D.G.), Amer. Math. Monthly 71 (1964), 385-391.

47. Certain embedding problems of semigroups (with Graham, N.), Proc. Japan Acad. 40 (1964), 8-13.

48. Examples of completely exclusive direct product, Portugal. Math. 23 (1964), 41-54.

49. Another proof of a theorem concerning the greatest semilattice-decomposition of a semigroup, Proc. Japan Acad. 40 (1964), 777-780.

50. The number of subsemigroups of certain semigroups (with Merkel, R.B.), Portugal. Math. 23 (1964), 183-191.

51. Semigroups in which all subsemigroups are left ideals (with Kimura, Naoki; Merkel, Rudolph), Canad. J. Math. 17 (1965), 52-62.

52. Report on attainability of systems of identities, Bull. Amer. Math. Soc. 71 (1965), 555-558.

53. Subsemigroups of completely 0 -simple semigroups, I. (with Chrislock, J.L.), Proc. Japan Acad. 41 (1965), 128-131.

54. Examples related to attainability of identities on lattices and rings (with Yaqub, F.M.), Math. Japon. 10 (1965), 35-39.

55. Semigroups with a maximal homomorphic image having zero (with Plemmons, R.J.), Proc. Japan Acad. 41 (1965), 681-685.

56. Non-negative integer valued functions on commutative groups, I. (with Biggs, R.; Sasaki, Morio), Proc. Japan Acad. 41 (1965), 564-569.

57. Completely 0 -simple subsemigroups of completely 0 -simple semigroups with finite structure groups (with Chrislock, J.L.), Math. Japon. 10 (1965), 91-100.

58. The theory of operations on binary relations, Trans. Amer. Math. Soc. 120 (1965), 343-358; Errata, ibid. 123 (1965), 273 and 134 (1968), 383.

59. Attainability of systems of identities on semigroups, J. Algebra 3 (1966), 261276.

60. On locally cyclic semigroups (with Levin, Richard), Proc. Japan Acad. 42 (1966), 376-379.

61. The lattice of congruences of locally cyclic semigroups (with Etterbeek, Wallace), Proc. Japan Acad. 42 (1966), 682-684.

62. Another proof of two decomposition theorems of semigroups (with Shafer, J.), Proc. Japan Acad. 42 (1966), 685-687.

63. Minimal or smallest relation of given type, Proc. Japan Acad. 42 (1966), 111114.

64. Semigroups connected with equivalence and congruence relations (with Dickinson, Robert), Proc. Japan Acad. 42 (1966), 688-692.

65. Notes on commutative Archimedean semigroups, I, II., Proc. Japan Acad. 42 (1966), 35-40; ibid. 42 (1966), 545-548.

66. Subdirect products of semigroups and rectangular bands (with Chrislock, J.L.), Bull. Amer. Math. Soc. 73 (1967), 637-638; Errata, ibid. 74 (1968), 628.

67. Decomposability of extension and its application to finite semigroups, Proc. Japan Acad. 43 (1967), 93-97. 
68. Power semigroups (with Shafer, J.), Math. Japon. 12 (1967), 25-32; Errata, ibid. 29 (1984), no. 4, 679.

69. Notes on groupoids and their automorphism groups, Proc. Japan Acad. 43 (1967), 843-846.

70. Maximal or greatest homomorphic images of given type, Canad. J. Math. 20 (1968), 264-271.

71. Construction of trees and commutative Archimedean semigroups, Math. Nachr. 36 (1968), 257-287.

72. On concentric semigroups, Proc. Japan Acad. 44 (1968), 868-870.

73. Note on homogeneous homomorphisms, Proc. Japan Acad. 44 (1968), 871872.

74. Semigroups satisfying $x y^{m}=y x^{m}=\left(x y^{m}\right)^{n}$ (with Mead, D.G.), Proc. Japan Acad. 44 (1968), 779-781.

75. On free contents, Proc. Japan Acad. 44 (1968), 865-867.

76. Notes on medial Archimedean semigroups without idempotent, Proc. Japan Acad. 44 (1968), 776-778.

77. Notes on subdirect products of semigroups and rectangular bands (with Chrislock, J.L.), Proc. Amer. Math. Soc. 20 (1969), 511-514.

78. Commutative semigroups whose lattice of congruence is a chain, Bull. Soc. Math. France 97 (1969), 369-380.

79. Semigroups satisfying identity $x y=f(x, y)$, Pacific J. Math. 31 (1969), 513521.

80. Note on finite commutative nil-semigroups (with Yamada, Miyuki), Portugal. Math. 28 (1969), 189-203.

81. The study of closets and free contents related to semilattice decomposition of semigroups, Semigroups (Proc. Sympos., Wayne State Univ. Detroit, Mich., 1968), pp. 221-260. Academic Press, New York, 1969.

82. Some contributions of computation to semigroups and groupoids, 1970 Computational Problems in Abstract Algebra (Proc. Conf., Oxford, 1967) pp. 229-261, Pergamon, Oxford.

83. Finite union of commutative power joined semigroups, Semigroup Forum 1 (1970), no. 1, 75-83.

84. Notes on finite left amenable semigroups, Proc. Japan Acad. 46 (1970), 217221.

85. Abelian groups and $R$-semigroups, Proc. Japan Acad. 46 (1970), 212-216.

86. Notes on commutative power joined semigroups (with Levin, Richard G.), Pacific J. Math. 35 (1970), 673-679.

87. Commutative Archimedean cancellative semigroups without idempotent, 1970 Séminaire P. Dubreil, M.-L. Dubreil-Jacotin, L. Lesieur et C. Pisot: 1969/70, Algèbre et Théorie des Nombres Fasc. 2, Exp. DG5, 19 pp. Secrétariat mathématique, Paris.

88. On commutative exclusive semigroups, Semigroup Forum 2 (1971), no. 2, 181187.

89. Positive rational semigroups and commutative power joined cancellative semigroups without idempotent (with Sasaki, Morio), Czechoslovak Math. J. 21(96) (1971), 567-576. 
90. Commutative semigroups with greatest group-homomorphism (with Hamilton, Howard B.), Proc. Japan Acad. 47 (1971), 671-675.

91. Theory of semigroups, Kyoritsu Publ., Tokyo, 1972 (Japanese).

92. Is minimal group congruence smallest? (with Hamilton, Howard B.), Semigroup Forum 4 (1972), 173-176.

93. On Putcha's theorem concerning semilattice of Archimedean semigroups, Semigroup Forum 4 (1972), 83-86.

94. Note on the greatest semilattice decomposition of semigroups, Semigroup Forum 4 (1972), 255-261.

95. Finiteness of lattice of congruences on commutative semigroups (with Nordahl, Tom E.), Semigroup Forum 4 (1972), 73-77.

96. Basic study of general products and homogeneous homomorphisms, I., Acta Sci. Math. (Szeged) 33 (1972), 31-44.

97. The study of commutative semigroups with greatest group-homomorphism (with Hamilton, Howard B.), Trans. Amer. Math. Soc. 173 (1972), 401-419.

98. On exponential semigroups, I. (with Shafer, John), Proc. Japan Acad. 48 (1972), 77-80.

99. Remark on the smallest semilattice congruence, Semigroup Forum 5 (1972/73), 277-282.

100. On exponential semigroups, II. (with Nordahl, Thomas E.), Proc. Japan Acad. 48 (1972), 474-478.

101. $N$-congruences of $N$-semigroups, J. Algebra 27 (1973), 11-30.

102. Finitely generated steady $N$-semigroups, Proc. Amer. Math. Soc. 41 (1973), 425-430.

103. Semilattice congruences viewed from quasi-orders, Proc. Amer. Math. Soc. 41 (1973), 75-79.

104. Finitely generated $N$-semigroup and quotient group (with Higgins, John C.), Proc. Japan Acad. 49 (1973), 323-327.

105. On the exponents of inverse semigroups, Proceedings of a Symposium on Inverse Semigroups and their Generalisations (Northern Illinois Univ. DeKalb, Ill., 1973), pp. 172-185. Northern Illinois Univ. DeKalb, Ill., 1973.

106. Survey of semilattice congruences on semigroups, Mini-Conference on Semigroup Theory (József Attila Univ. Szeged, 1972), pp. 47-57. József Attila Univ. Szeged, 1973.

107. Note on attainability of identities on bands, J. Algebra 28 (1974), 1-9.

108. Complementary semigroups and exponent semigroups of order bounded groups, Math. Nachr. 59 (1974), 17-34.

109. Irreducible $N$-semigroups, Math. Nachr. 63 (1974), 71-88.

110. Basic study of $N$-semigroups and their homomorphisms, Semigroup Forum 8 (1974), no. 1, 21-50.

111. Basic study of $N$-semigroups and their homomorphisms, II., Semigroup Forum 10 (1975), no. 3, 250-261.

112. Semi-closure mappings and cofinal chains, Portugal. Math. 34 (1975), no. 1-2, 111-117.

113. Quasi-orders, generalized Archimedeanness and semilattice decompositions, Math. Nachr. 68 (1975), 201-220. 
114. Commutative cancellative semigroups without idempotents (with Hamilton, H.B.; Nordahl, T.E.), Pacific J. Math. 61 (1975), no. 2, 441-456.

115. Putcha's problem on maximal cancellative subsemigroups, Proc. Japan Acad. 52 (1976), no. 3, 91-94.

116. Homomorphisms of commutative cancellative semigroups into nonnegative real numbers (with Putcha, Mohan S.), Trans. Amer. Math. Soc. 221 (1976), no. 1, 147-157.

117. Commutative Archimedean cancellative idempotent-free semigroups with an infinite cyclic group as structure group, J. Algebra 45 (1977), no. 2, 343-390.

118. Completely semisimple inverse Delta-semigroups admitting principal series, Pacific J. Math. 68 (1977), no. 2, 515-525.

119. $N$-semigroups and their translation semigroups, J. Austral. Math. Soc. Ser. A 24 (1977), no. 2, 184-202.

120. Commutative subarchimedean semigroups, Seminar on semigroup theory (Res. Inst. Math. Sci., Kyoto Univ. Kyoto, 1977), Sûrikaisekikenkyûsho Kōkyûroku No. 292 (1977), 98-126.

121. Quasi-order preserving homomorphisms of commutative semigroups into the non-negative reals (with Kobayashi, Yuji), Math. Japon. 22 (1977), no. 3, 267285.

122. Notes on translational hulls of completely 0-simple semigroups, Proceedings of the First Symposium on Semigroups (Shimane Univ. Matsue, 1977), pp. 73-83. Shimane University, Matsue, 1977; Errata, ibid. Proceedings of the 2nd Symposium on Semigroups (Tokyo Gakugei Univ. Tokyo, 1978), p. 80, Tokyo Gakugei Univ. Tokyo, 1978.

123. Recent results and problems on commutative Archimedean semigroups, Proceedings of the 2nd Symposium on Semigroups (Tokyo Gakugei Univ. Tokyo, 1978), pp. 69-79, Tokyo Gakugei Univ. Tokyo, 1978.

124. Maximal cancellative subsemigroups and sub-Archimedeanness (with Gale, D.), Algebraic theory of semigroups (Proc. Sixth Algebraic Conf., Szeged, 1976), pp. 147-154, Colloq. Math. Soc. János Bolyai, 20, North-Holland, Amsterdam-New York, 1979.

125. Identities $E-2$ and exponentiality (with Clarke, J.; Pfiefer, R.), Proc. Japan Acad. Ser. A Math. Sci. 55 (1979), no. 7, 250-251.

126. Finite semigroups whose congruences form chains, Proceedings of the Conference on Semigroups in Honor of Alfred H. Clifford (Tulane Univ. New Orleans, La., 1978), pp. 94-116, Tulane Univ. New Orleans, La., 1979.

127. Extensions of partially ordered Abelian groups, Proc. Japan Acad. Ser. A Math. Sci. 56 (1980), no. 3, 130-132.

128. Structure groups of nontorsion Abelian groups, Math. Japon. 25 (1980), no. 1, 101-109.

129. Permutation groups and ideal extensions, Proceedings of the 3rd Symposium on Semigroups (Inter-Univ. Sem. House of Kansai, Kobe, 1979), pp. 83-103, Osaka Univ. Osaka, 1980.

130. A theorem on commutative cancellative Archimedean idempotent-free semigroups (with Gale, Deborah), Simon Stevin 54 (1980), no. 3-4, 233-240. 
131. Homomorphisms of free $E-n$ algebraic systems, Proceedings of the 4th Symposium on Semigroups (Yamaguchi Univ. Yamaguchi, 1980), pp. 103-111, Yamaguchi Univ. Yamaguchi, 1980.

132. Semigroups whose congruences form a chain and which are extensions of congruence-free semigroups, Semigroups (Proc. Conf., Monash Univ. Clayton, 1979), pp. 85-102, Academic Press, New York-London, 1980.

133. Notes on semirings whose multiplicative semigroups are groups, Proceedings of the 5th Symposium on Semigroups (Sakado, 1981), pp. 56-66, Jōsai Univ. Sakado, 1981.

134. Strongly reversible groups, Proc. Amer. Math. Soc. 84 (1982), no. 3, 325-330.

135. Semilattice indecomposable semigroups with a unique idempotent, Semigroup Forum 24 (1982), no. 1, 77-82.

136. Free $E-m$ groups and free $E-m$ semigroups, Proc. Amer. Math. Soc. 84 (1982), no. 3, 318-324.

137. Nil-orders of commutative nil-bounded semigroups, Semigroup Forum 24 (1982), no. 2-3, 255-262.

138. Finite inverse perfect semigroups and their congruences (with Hamilton, Howard), J. Austral. Math. Soc. Ser. A 32 (1982), no. 1, 114-128.

139. Commutative cancellative semigroups with nontrivial homomorphisms into nonnegative real numbers, J. Algebra 76 (1982), no. 1, 25-41.

140. Binary systems all subsets of which are recognizable, J. Algebra 76 (1982), no. $1,42-83$.

141. On semigroups of subsets of semigroups, II. Idempotents in power semigroups of finite 0 -simple semigroups, Proceedings of the 7 th symposium on semigroups (Tokyo, 1983), 12-19, Tokyo Gakugei Univ. Tokyo, 1983.

142. Power semigroups of rectangular groups, Math. Japon. 29 (1984), no. 4, 671678.

143. On chains whose power semigroups are lattices, Semigroup Forum 30 (1984), no. $35-40$.

144. On the power semigroup of the group of integers, Proc. Japan Acad. Ser. A Math. Sci. 60 (1984), no. 10, 388-390.

145. Archimedean components of the power semigroup of the infinite cyclic group, Proceedings of the 8th symposium on semigroups (Matsue, 1984), 62-70, Shimane Univ. Matsue, 1985.

146. $S$-indecomposable semigroups generated by two elements (with Sasaki, Morio), Proceedings of the 9th symposium on semigroups and related topics (Naruto City, 1985), 3-5, Naruto Univ. Teacher Ed., Naruto-shi, 1985.

147. On the power semigroup of a finite 0 -simple semigroup-rectangle $J$-classes, Proceedings of the 9th symposium on semigroups and related topics (Naruto City, 1985), 48-54, Naruto Univ. Teacher Ed., Naruto-shi, 1985.

148. Algebraic systems associated with involuted regular semigroups, Semigroups (Szeged, 1981), 471-488, Colloq. Math. Soc. János Bolyai, 39, North-Holland, Amsterdam, 1985.

149. Isomorphism problem of power semigroups of completely 0-simple semigroups, J. Algebra 98 (1986), no. 2, 319-361. 
150. Archimedean components of the power semigroup of the group of rational numbers (with Sasaki, Morio; Spake, Reuben), Proceedings of the 10th symposium on semigroups (Sakado, 1986), 31-35, Jōsai Univ. Sakado, 1987.

151. On the power semigroup of a finite 0-simple semigroup-nonrectangular $J$ classes, Proceedings of the 10th symposium on semigroups (Sakado, 1986), 36-44, Jōsai Univ. Sakado, 1987.

152. Identities satisfied by power semigroups (with Yamaoka, Kenya), Proceedings of the 10th symposium on semigroups (Sakado, 1986), 45-51, Jōsai Univ. Sakado, 1987.

153. On the recent results in the study of power semigroups, Semigroups and their applications (Chico, Calif., 1986), 191-200, Reidel, Dordrecht, 1987.

154. Boundly factorizable $S$-indecomposable semigroups generated by two elements (with Sasaki, Morio), Proc. Japan Acad. Ser. A Math. Sci. 63 (1987), no. 8, 315317.

155. Note on Boolean matrices and semigroups (with Yamaoka, Kenya), Proceedings of the 11th Symposium on Semigroups (Kyoto, 1987), 19-24, Reikichi Yoshida, Matsubara, 1987.

156. The 0-minimal ideal of the global of a combinatorial completely 0-simple semigroup (with Yamaoka, Kenya), Proc. Japan Acad. Ser. A Math. Sci. 63 (1987), no. 9, 364-366.

157. The zero containing property of isomorphisms of finite power semigroups (with Yamaoka, Kenya), Proceedings of the 12th Symposium on Semigroups (Tokushima, 1988), 28-33, Tokushima Univ. Tokushima, 1988.

158. Note on finite power semigroups (with Yamaoka, Kenya), Proceedings of the 13th Symposium on Semigroups (Kyoto, 1989), 11-15, Kyoto Sangyo Univ. Kyoto, 1990.

159. The regular $J$-class of the power semigroup of a completely 0 -simple semigroup, Math. Nachr. 149 (1990), 7-27.

160. Notes on construction of subdirect products of groups of integers (with Dickinson, Robert), Proceedings of the 15th Symposium on Semigroups (Matsue, 1991), 79-87, Shimane Univ. Matsue, 1992.

161. Notes on construction of subdirect products of groups of integers II., Proceedings of the 16th Symposium on Semigroups, Languages and their Related Fields (Funabashi, 1992), 15-20, Toho Univ. Funabashi, 1992.

162. Construction of subdirect products of groups of integers, I. (with Dickinson, Robert), Pure Math. Appl. Ser. A 3 (1992), no. 3-4, 161-183 (1993).

163. Notes on semigroups of standard subdirect products of groups of integers (with Dickinson, Robert), Proceedings of the 18th Symposium on Semigroups, Languages and their Related Fields (Koganei, 1994), 23-29, Tokyo Gakugei Univ. Tokyo, 1994.

164. The study of the semigroup of subdirect products of the groups of integers, Proceedings of the 19th Symposium on Semigroups, Languages and their Related Fields (Matsue, 1995), 60-64, Shimane Univ. Matsue, 1995.

165. Note on $S$-indecomposable semigroups having zero as a unique idempotent, Proceedings of the Third Symposium on Algebra, Languages and Computation (Osaka, 1999), 68-71, Shimane Univ. Matsue, 2000. 
166. Note on $S$-indecomposable semigroups having zero as a unique idempotent, II., Proceedings of the 4th Symposium on Algebra, Languages and Computation (Osaka, 2000), 77-82, Osaka Prefect. Univ. Sakai, 2001.

167. Finitely generated idempotent-free semilattice-indecomposable semigroups with relations I., Algebraic semigroups, formal languages and computation (Japanese) (Kyoto, 2001). Sûrikaisekikenkyûsho Kōkyûroku No. 1222 (2001), 86-89.

The authors wish to acknowledge the help and advice of Laszlo Marki, Norman Reilly, and Mohan Putcha. 